\title{
Comparing Oxidative Stress Status Among Iranian Males and Females with Malignant and Non-malignant Thyroid Nodules
}

\author{
Bita Faam ${ }^{1}$, Ata A Ghadiri ${ }^{1,2}$, Mohammad Ali Ghaffari ${ }^{1}$, Mehdi Totonchi ${ }^{3}$ and Layasadat Khorsandi ${ }^{1,4,}{ }^{*}$ \\ ${ }^{1}$ Cellular \& Molecular Research Center, Medical Basic Sciences Research Institute, Ahvaz Jundishapur University of Medical Sciences, Ahvaz, Iran \\ ${ }^{2}$ Immunology, School of Medicine, Ahvaz Jundishapur University of Medical Sciences, Ahvaz, Iran \\ ${ }^{3}$ Department of Endocrinology and Female Infertility, Reproductive Biomedicine Research Center, Royan Institute for Reproductive Biomedicine, Academic Center for \\ Education, Culture and Research (ACECR), Tehran, Iran \\ ${ }^{4}$ Department of Anatomical Sciences, School of Medicine, Ahvaz Jundishapur University of Medical Sciences, Ahvaz, Iran \\ "Corresponding author: Department of Anatomical Sciences, School of Medicine, Ahvaz Jundishapur University of Medical Sciences, Ahvaz, Iran. Email: \\ khorsandi_cmrc@yahoo.com
}

Received 2020 May 23; Revised 2020 December 16; Accepted 2021 January 07.

\begin{abstract}
Background: Oxidative stress is commonly accrued in thyroid tissue during hormone synthesis.

Objectives: We aimed to examine oxidative stress in patients with thyroid cancer, benign thyroid nodules, and healthy individuals. Methods: In this study, 138 individuals were involved. Among the selected participants, 108 had thyroid nodules, including 30 papillary thyroid cancer (PTC), 30 follicular thyroid cancer (FTC), six anaplastic thyroid cancer (ATC), 12 medullary thyroid cancer (MTC), and 30 benign nodules. In addition, 30 individuals were selected as a healthy control group. The levels of total antioxidant capacity (TAC) and total oxidant status (TOS) of thyroid tissue were measured using the ELISA method, and the oxidative stress index (OSI) was calculated.

Results: The TAC level was significantly lower in MTC and FTC subtypes than in controls. The TOS level was considerably higher in the MTC group than in the control and benign nodule groups. The TOS level was not changed in other groups. The OSI was considerably higher in MTC and FTC subtypes. The TAC and OSI in benign nodules were significantly lower and higher than those of controls, respectively. The OSI was higher in female patients than in males.

Conclusions: The OSI can not be considered a diagnostic biomarker for benign nodules and MTC. The diverse oxidative stress status between genders may be related to the elevated cancer incidence in females.
\end{abstract}

Keywords: Oxidative Stress, Oxidative Marker, Thyroid Nodule, Cancer

\section{Background}

Thyroid cancer accounts for approximately $1 \%$ of all types of human cancer. The incidence of this cancer is increasing worldwide $(1,2)$. Over $95 \%$ of thyroid cancers have different neoplastic phenotypes, including papillary thyroid cancer (PTC), follicular thyroid cancer (FTC), and anaplastic thyroid cancer (ATC) (3). medullary thyroid cancer (MTC) is a rare neoplasm of calcitonin-secreting thyroid cells, which accounts for $5 \%-10 \%$ of all thyroid cancers (4).

Oxidative stress is a kind of imbalance between reactive oxygen and nitrogen species, which results in the antioxidant defense. Free radicals can disturb important biomolecules such as DNA, lipids, and proteins (5). The redox imbalance can induce chronic diseases and cancer by affecting the oxygen species in molecular pathways (6). Although some oxidants contribute to mutation and growth, excessive oxidative stress can slow proliferation and dam- age cancerous cells $(7,8)$.

The oxidative status depends on endogenous enzymatic factors and exogenous factors such as diet, medication, and lifestyle (9-11). Thyroid hormones play important roles in regulating oxidative metabolism via adjusting the synthesis and degradation of enzymatic antioxidants such as catalase and superoxide dismutase and non-enzymatic antioxidants, including vitamin C and E (12). The oxidative stress is commonly accrued in thyroid tissue during utilizing $\mathrm{H}_{2} \mathrm{O}_{2}$ for thyroxin synthesis (13). However, only a few epidemiological studies have investigated the oxidative stress status in thyroid cancer (14).

Evaluating the levels of total oxidant status (TOS) and total antioxidant capacity (TAC) is an improved way compared to assessing oxidants and antioxidants separately, which is expensive and time-consuming. 


\section{Objectives}

Therefore, in the present work, we evaluated TOS, TAC, and oxidative stress index (OIS) in thyroid tissues of patients with thyroid cancer, benign thyroid nodules, and healthy control individuals in an Iranian population.

\section{Methods}

\subsection{Study Population}

The current work was carried out in an Iranian population of Erfan Grand Hospital (Tehran). From 2017 to 2019, 138 selected individuals ( 98 females and 40 males) aged 27 - 74 years participated in the current work. The thyroid nodules of patients were detected by clinical examination. Fine needle aspiration (FNA) was performed for all individuals. Thyroidectomy was done for patients with malignant or repetitive indeterminate nodules. Among the selected participants, 108 had thyroid nodules, including 30 PTC, 30 FTC, 6 ATC, 12 MTC, and 30 benign nodules (Table 1). In addition, 30 individuals were selected to form a healthy control group. The Institute Ethics Committee (grant number: ajums.REC.1393.151) approved this work, and informed consent was obtained for all biopsy samples.

\subsection{Tissue Samples}

Thyroid tissues were collected and assessed by an expert pathologist. Thyroid tissues were promptly stored at $-80^{\circ} \mathrm{C}$ until use. They were homogenized and maintained in the RIPA buffer (on ice) for 30 min and then sonicated. The samples were centrifuged at $10,000 \mathrm{~g}\left(10 \mathrm{~min}\right.$ at $\left.4^{\circ} \mathrm{C}\right)$, and the supernatants were stored at $-20^{\circ} \mathrm{C}$ until use.

\subsection{Total Antioxidant Capacity}

Total Antioxidant Capacity (TAC) was measured calorimetrically by the TAC kit (ZellBio GmbH, Germany). This assay is based on the reduction of $\mathrm{Fe}^{+3}$ to $\mathrm{Fe}^{+2}$, which can combine with phenanthrene to form a colored compound. The TAC was presented as mmol TROLOX Eq/L.

\subsection{Total Oxidant Status}

The thyroid tissue total oxidant status (TOS) was measured calorimetrically using the TOS kit (ZellBio GmbH assay kit). The results were expressed in $\mu \mathrm{mol}_{2} \mathrm{O}_{2}$ equivalent $/ \mathrm{L}(\mu \mathrm{mol} \mathrm{H} 2 \mathrm{O} 2 \mathrm{Eq} / \mathrm{L})$. The TOS assay kit could determine TOS in biological samples with $0.5 \mu$ M sensitivity.

\subsection{Oxidative Stress Index}

The TOS to TAC ratio was regarded as the oxidative stress index (OSI). The unit of TAC, mmol Trolox Eq/L, was converted to mmol Trolox Eq/L, and OSI was calculated as follows $(14,15)$ : OSI $=$ TOS $/$ TAC $\times 100$

\subsection{Thyroid Profiles}

After overnight fasting, blood samples were collected. Approximately $5 \mathrm{ml}$ of blood was collected for thyroid hormones analysis. After incubating the samples at room temperature for $10 \mathrm{~min}$, they were centrifuged at 3,000 rpm for eight minutes, and the obtained sera were maintained at $80^{\circ} \mathrm{C}$. The serum levels of thyroxine (T4), triiodothyronine (T3), and thyrotropic-stimulating hormone (TSH) were determined using available commercial kits (DiaSorin, Italy).

\subsection{Statistical Analysis}

All statistical analyses were performed using SPSS 21.0 software. The normal distribution of data and homogeneity of variances were checked using the Shapiro-Wilk test and Levene's test, respectively. A one-way analysis of variance (ANOVA), followed by Kruskal-Wallis post hoc pairwise comparisons, was used to analyze the data. All data were presented as mean \pm standard deviation (SD). A Pvalue $<0.05$ was considered significant.

\section{Results}

The TAC was considerably lower in patients with benign nodules than in normal patients. The TAC level of PTC and ATC subtypes was not considerably changed when compared to the control group, but it was considerably increased compared to the benign group. The TAC level was considerably lower in FTC and MTC patients than in the control group. The TOS level was considerably higher in the MTC group than in the control and benign nodule groups. In the other subtypes of thyroid cancer and benign groups, the TOS level was not considerably changed when compared to the control group. The OSI level was considerably higher in patients with benign nodules than in the normal group. The OSI level of MTC and FTC subtypes was considerably higher than that of the control and benign groups. The OSI of other subtypes was not considerably changed when compared to the control group (Figure 1). The OSI was higher in female patients with thyroid cancer or benign nodules than in male patients (Table 1). The thyroid hormone levels were not significantly changed in patients with benign and cancer subtypes when compared to the control group (Figure 2).

There were no correlations between thyroid hormone levels and oxidative stress status in different groups. In addition, there were no correlations between the age of patients and oxidative/antioxidative parameters (Table 2). 
Faam B et al.

\begin{tabular}{|c|c|c|c|c|}
\hline Groups (n) & $\operatorname{Sex}(N)$ & TAC, mmol Trolox Eq/L & $\mathrm{TOS}, \mathrm{mmol} \mathrm{H}_{2} \mathrm{O}_{2} \mathrm{Eq} / \mathrm{L}$ & OSI, Arbitrary Unit \\
\hline \multirow{2}{*}{ Normal (30) } & Male (12) & $1.11 \pm 0.24$ & $15.45 \pm 2.38$ & $1.39 \pm 0.19$ \\
\hline & Female (18) & $1.21 \pm 1.09$ & $19.92 \pm 4.51$ & $1.64 \pm 0.22$ \\
\hline Benign (30) & Male (9) & $0.17 \pm 0.58$ & $16.45 \pm 3.51$ & $2.83 \pm 0.27$ \\
\hline \multirow{2}{*}{ PTC (30) } & Male (8) & $1.58 \pm 0.21$ & $17.05 \pm 0.13$ & $1.07 \pm 0.15$ \\
\hline & Female (22) & $0.96 \pm 0.18$ & $22.36 \pm 4.39$ & $2.32 \pm 0.24^{*}$ \\
\hline \multirow{2}{*}{ FTC (30) } & Male (5) & $0.43 \pm 0.11$ & $10.14 \pm 2.4$ & $2.35 \pm 0.31$ \\
\hline & Female (25) & $0.45 \pm 0.08$ & $18.85 \pm 2.9$ & $4.18 \pm 0.43^{*}$ \\
\hline \multirow{2}{*}{ MTC (12) } & Male (4) & $0.19 \pm 0.06$ & $14.5 \pm 3.11$ & $7.63 \pm 1.45$ \\
\hline & Female (8) & $0.38 \pm 0.08^{*}$ & $40.7 \pm 5.76^{* *}$ & $10.71 \pm 2.18$ \\
\hline \multirow{2}{*}{$\operatorname{ATC}(6)$} & Male (2) & $1.8 \pm 0.25$ & $17.1 \pm 2.82$ & $0.91 \pm 0.18$ \\
\hline & Female (4) & $0.83 \pm 0.12$ & $20.1 \pm 3.92$ & $2.42 \pm 0.29^{*}$ \\
\hline
\end{tabular}

Abbreviations: ATC, anaplastic thyroid cancer; FTC, follicular thyroid cancer; MTC, medullary thyroid cancer; OSI, oxidative stress index; PTC, papillary thyroid cancer TAC, total antioxidant capacity; TOS, total oxidant status.

${ }^{\mathrm{a}}$ Values are expressed as mean $\pm \mathrm{SD}$

${ }^{\mathrm{b}}, \mathrm{P}<0.05,{ }^{* *}, \mathrm{P}<0.01 ;{ }^{*}$, indicates comparisons with male patients.

Table 2. Oxidative Stress Index in Normal, Benign, and Total Thyroid Cancer Patients $^{\mathrm{a}}$

\begin{tabular}{lcc}
\hline Groups (N) & Age, Years & OSI, Arbitrary Unit \\
\hline Normal (30) & $52.51 \pm 5.7$ & $1.52 \pm 0.29$ \\
Benign (30) & $49.15 \pm 6.9$ & $6.26 \pm 2.27^{\mathrm{b}}$ \\
\hline Thyroid cancer (78) & $53.5 \pm 2.12$ & $4.1 \pm 0.88$ \\
\hline
\end{tabular}

Abbreviation: OSI, oxidative stress index.

${ }^{\mathrm{a}}$ Values are expressed as mean $\pm \mathrm{SD}$.

${ }^{\mathrm{b}} \mathrm{P}<0.001$

\section{Discussion}

In humans, the complex antioxidant system can counteract free radicals and reactive oxygen species. This system includes different antioxidant substances such as enzymatic and non-enzymatic antioxidants, which may be known or unknown (16). Therefore, it is essential to determine the total oxidant and antioxidant levels to evaluate the oxidative stress status. Previous studies have examined the role of various individuals' oxidative or anti-oxidative molecules in thyroid cancer.

Our results showed that oxidative stress status in patients with thyroid cancer is different from that of individuals with benign nodules and healthy individuals. Accordingly, TAC was lower in thyroid cancer patients, whereas OSI was higher in these patients. Akinci et al. compared the antioxidant enzyme activities between 43 patients with thyroid cancer and 43 control individuals. They reported that the superoxide dismutase level did not alter in thyroid cancer patients. However, malondialdehyde and antioxidant glutathione peroxidase changed with thyroid cancer (13). Among the cancer subtypes in this study, FTC and MTC groups showed a lower TAC level than other cancer subtypes. In mice with aggressive FTC, the upregulation of superoxide dismutase (SOD) reduced FTC proliferation, whereas SOD deficiency enhanced tumor growth (17). Hence, decreasing TAC may involve in the pathogenesis of FTC. In a study by Miri and Mohammadi (18), TAC and catalase levels were decreased in individuals with MTC. They suggested that oxidative stress may be associated with MTC or enhance the risk of developing the disease. Another study in 2016 in an Iranian population with MTC showed that serum TAC, catalase (CAT), and glutathione (GSH) levels were lower in MTC patients. Accordingly, oxidant/antioxidant imbalance could be associated with an increased risk of medullary thyroid cancer (19).

In our study, the ATC group showed no significant change in OSI and TAC levels. In the study by Wang et al. (14), the OSI value was not changed in ATC patients. Another study by Erdamar et al. (20) evaluated the oxidative markers in the serum of 41 patients with multinodular goiter and PTC. They examined malondialdehyde, selenium, total superoxide dismutase, and glutathione peroxidase in selected patients. Accordingly, superoxide dismutase, glutathione peroxidase, and selenium levels were found to be lower and malondialdehyde to be higher in both nodules and cancerous tissues when compared to patients with nontoxic multinodular goiter. They showed that lipid peroxidation was increased, and the enzymatic free-radical de- 

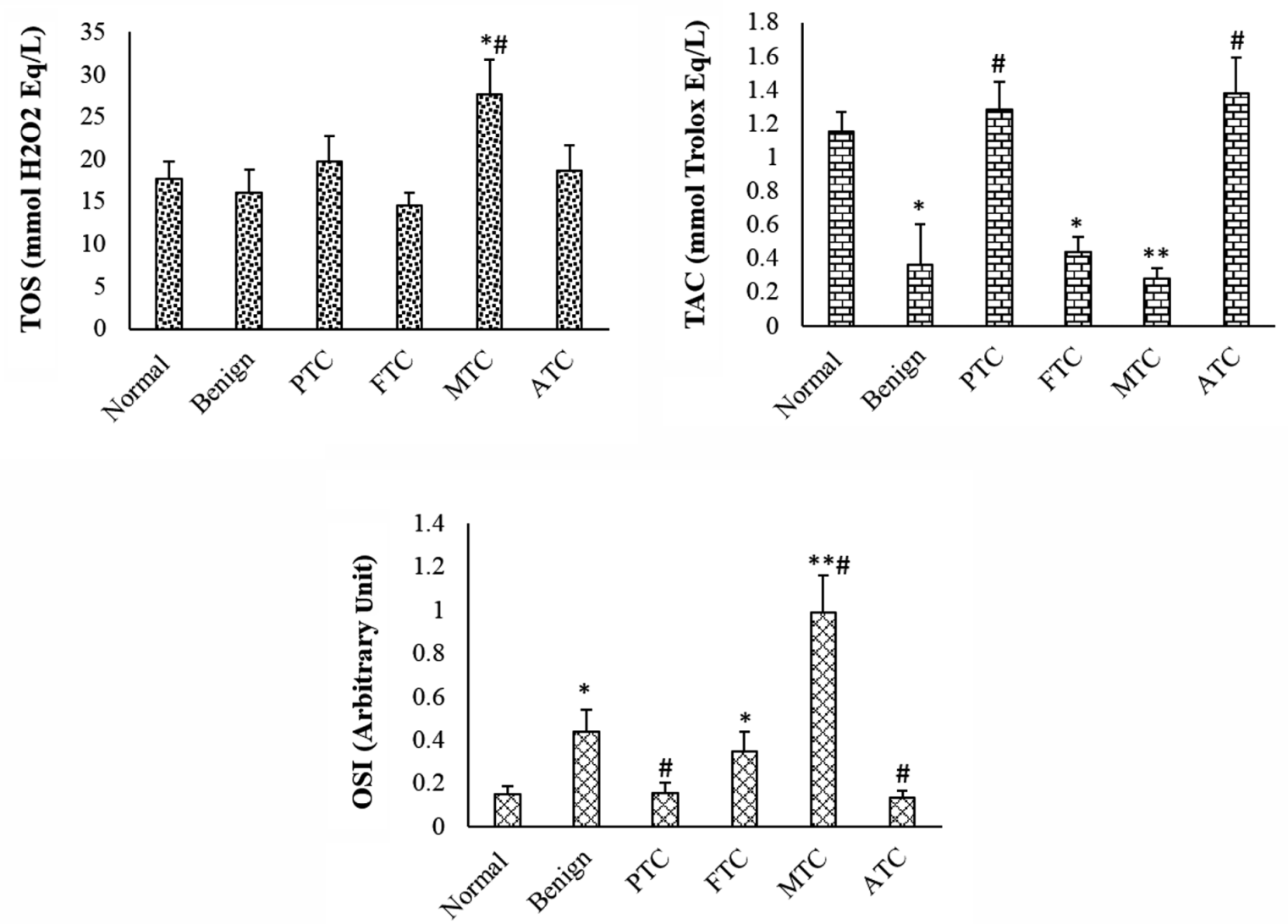

Figure 1. Total oxidant status, total antioxidant capacity, and oxidative stress index in different groups. Values are presented as mean $\pm S D$. ${ }^{*}, \mathrm{P}<0.05$, ${ }^{* *}, \mathrm{P}<0.01, \mathrm{P}<0.05$ * and \#, indicate comparisons with normal and benign groups. TOS, total oxidant status; TAC, total antioxidant capacity; OSI, oxidative stress index; PTC, papillary thyroid cancer; FTC, follicular thyroid cancer; MTC, medullary thyroid cancer; ATC, anaplastic thyroid cancer.

fense system was impaired in patients with multinodular goiter and PTC (20). In the study by Famil Sagharchian et al. (21), serum TAC and malondialdehyde contents were low and high in PTC individuals, respectively. Lassoued et al. (22) showed that oxidative status was highly disturbed in PTC patients. However, in the present work, OSI and TAC were not changed in PTC patients.

The opposite results may be because the levels of oxidative stress markers in blood samples are different from those in thyroid tissue samples. In addition, genetic variants may be influenced by oxidative stress markers in different individuals. Moreover, by considering only some of the antioxidant enzymatic activities, the role of the oxidative stress index could not be conducted, which was done in our study by measuring TOS and TAC levels. In 2011, for the first time, Wang et al. (14) recommended measuring the OSI in individuals with thyroid cancer. They reported that TAC and TOS levels were significantly different between healthy and thyroid cancer groups. However, they did not find any difference in the serum concentrations of TAC, TOS, and OSI between carcinoma subtypes (14). Nevertheless, in our study, tissue concentrations of TAC, TOS, and OSI were considerably changed in MTC and FTC cancer subtypes.

As the results showed, the OSI was higher in females with thyroid cancer than in males. Previous studies also showed that thyroid cancer is more prevalent in females than in males. High levels of $\mathrm{H}_{2} \mathrm{O}_{2}$ are generated in the thyroid gland that regulates cell survival, proliferation, migration, and death. The cellular oxidant-antioxidant imbalance may contribute to the more incidence of thyroid cancer among females. In addition, $17 \beta$-estradiol administration was observed to increase $\mathrm{H}_{2} \mathrm{O}_{2}$ generation in a thyroid cell line ( $\mathrm{PCCL}_{3}$ cells) (22). In the process of thyroid cancer, estrogen receptors may be developed and proliferated (23). Fortunato et al. (24) showed that the thyroid antioxidant defense of female rats was weaker than that of male rats. In our results, TAC levels were also lower in female pa- 

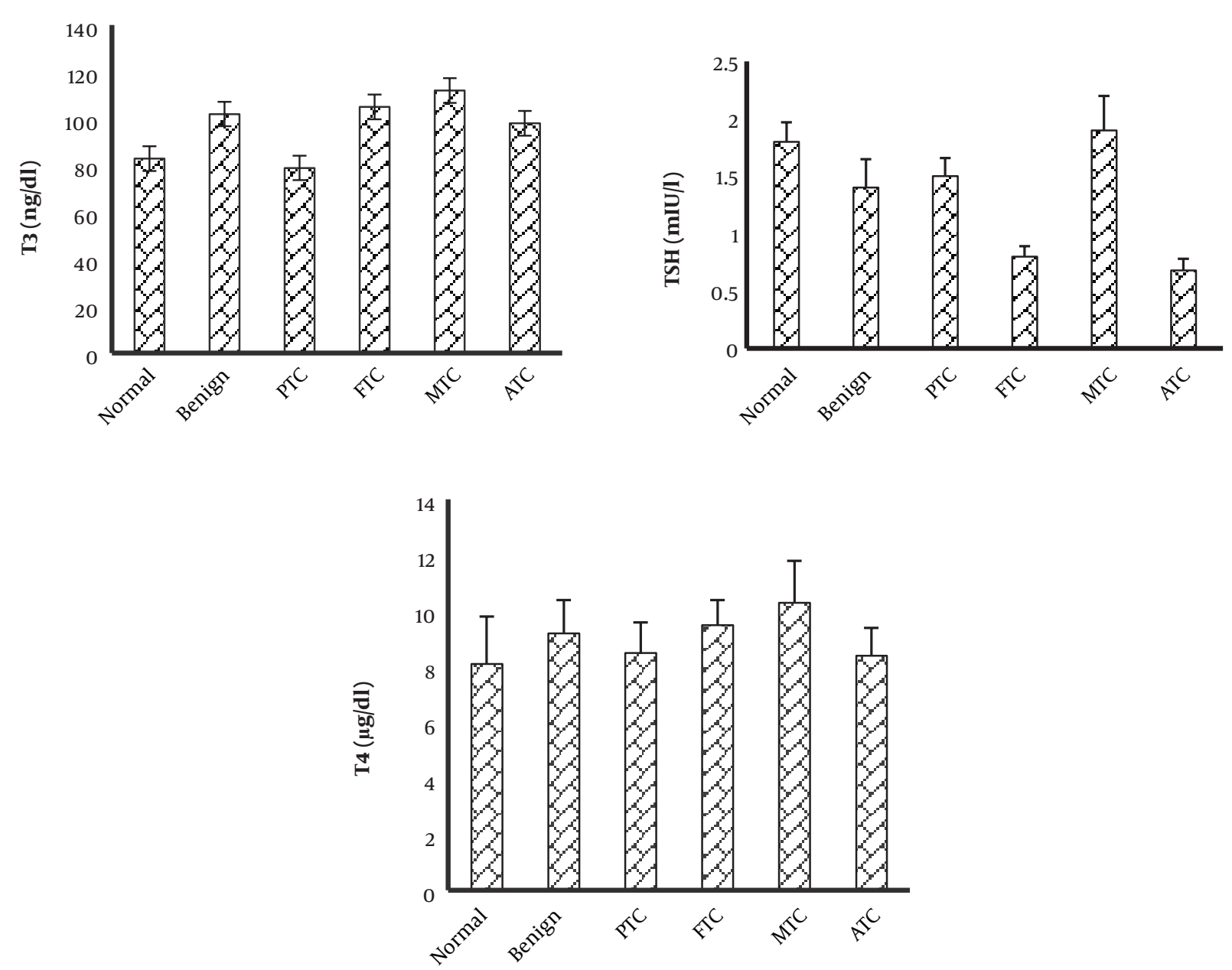

Figure 2. Thyroid hormone levels in different groups. Values are presented as mean \pm SD. All P-values are more than 0.05. PTC, papillary thyroid cancer; FTC, follicular thyroid cancer; MTC, medullary thyroid cancer; ATC, anaplastic thyroid cancer.

tients with thyroid cancer than in male patients. Besides, benign nodules showed a high level of TAC. In contrast, in the study by Stanley et al. (25), the oxidant-antioxidant balance was undisturbed in benign thyroid tumors, whilst in malignant tumors, the balance was altered.

Interestingly, the antioxidant system has a bidirectional role in tumorigenesis. In benign thyroid nodules of rats, the SOD level was enhanced but gradually diminished as cancer cells progressed towards a more de-differentiated phenotype (26). In mice, SOD overexpression elevated the burden of benign tumors but suppressed the progression of FTC. Hence, antioxidant/oxidant imbalance may contribute to the conversion of normal thyroid tissue to benign tumors.

Thyroid hormones play an important role in the generation of free radicals. Thyroid hormones modulate the pro- duction and degradation of non-enzymatic and enzymatic antioxidants (27). However, in this study, thyroid hormone levels of benign and all cancer subtypes were not considerably changed when compared to control individuals. In the study by Wang et al., the oxidative stress value was not correlated with the hormone profile (14).

\subsection{Conclusions}

This study showed that the OSI could not be considered a valuable prognostic biomarker because it was increased in MTC and individuals with benign nodules. The role of this index in differentiating various types of this cancer is debatable. The difference in the OSI between cancer subtypes indicates that oxidative stress may be more involved in the pathogenesis of MTC and FTC than in other subtypes. The diverse oxidative stress status between genders may be 
a critical factor in explaining the elevated cancer incidence in females.

\section{Acknowledgments}

The authors wish to acknowledge Ms. Niloofar Shiva for critical editing for English grammar and syntax and Ms. Parvaneh Arbab for critical laboratory support.

\section{Footnotes}

Authors' Contribution: Guarantor of integrity of the entire study: Layasadat Khorsandi. Study concept and design: Bita Faam and Mohammad Ali Ghaffari. Literature research: Ata A Ghadiri and Bita Faam. Analysis and interpretation of data: Mehdi Totonchi and Bita Faam. Clinical studies: Mehdi Totonchi and Mohammad Ali Ghaffari. Statistical analysis: Layasadat Khorsandi and Bita Faam. Drafting of the manuscript: Bita Faam and Mohammad Ali Ghaffari. Critical revision of the manuscript for important intellectual content: Layasadat Khorsandi.

Conflict of Interests: There is no conflict of interest for authors.

Ethical Approval: The ethical approval code was ajums.REC.1393.151 (behsan.ajums.ac.ir).

Funding/Support: This study was supported by a grant (code: CMRC-122) from Ahvaz Jundishapur University of Medical Sciences, awarded to Bita Faam for a PhD thesis.

Informed Consent: Informed consent forms were completed by the participants.

\section{References}

1. Faam B, Ghaffari MA, Ghadiri A, Azizi F. Epigenetic modifications in human thyroid cancer. Biomed Rep. 2015;3(1):3-8. doi: 10.3892/br.2014.375. [PubMed: 25469237]. [PubMed Central: PMC4251143].

2. Goodarzi E, Moslem A, Feizhadad H, Jarrahi AM, Adineh HA, Sohrabivafa $\mathrm{M}$, et al. Epidemiology, incidence and mortality of thyroid cancer and their relationship with the human development index in the world: an ecology study in 2018. Adv Hum Biol. 2019;9(2):162.

3. Asa SL. The Current Histologic Classification of Thyroid Cancer. Endocrinol Metab Clin North Am. 2019;48(1):1-22. doi: 10.1016/j.ecl.2018.10.001. [PubMed: 30717895].

4. Sheikholeslami S, Zarif Yeganeh M, Hoghooghi Rad L, Golab Ghadaksaz H, Hedayati M. Haplotype Frequency of G691S/S904S in the RET Proto-Onco-gene in Patients with Medullary Thyroid Carcinoma. Iran J Public Health. 2014;43(2):235-40. [PubMed: 26060748]. [PubMed Central: PMC4450692].

5. Sadati Zarrini A, Moslemi D, Parsian H, Vessal M, Mosapour A, Shirkhani Kelagari Z. The status of antioxidants, malondialdehyde and some trace elements in serum of patients with breast cancer. Caspian JIntern Med. 2016;7(1):31-6. [PubMed: 26958330]. [PubMed Central: PMC4761120].
6. Hecht F, Cazarin JM, Lima CE, Faria CC, Leitao AA, Ferreira AC, et al Redox homeostasis of breast cancer lineages contributes to differential cell death response to exogenous hydrogen peroxide. Life Sci. 2016;158:7-13. doi: 10.1016/j.lfs.2016.06.016. [PubMed: 27328417].

7. Schumacker PT. Reactive oxygen species in cancer: a dance with the devil. Cancer Cell. 2015;27(2):156-7. doi: 10.1016/j.ccell.2015.01.007. [PubMed: 25670075].

8. Coughlin SS. Oxidative Stress, Antioxidants, Physical Activity, and the Prevention of Breast Cancer Initiation and Progression. J Environ Health Sci.2018;4(2):55-7. doi:10.15436/2378-6841.18.2013. [PubMed: 30957018]. [PubMed Central: PMC6449844].

9. Costa A, Scholer-Dahirel A, Mechta-Grigoriou F. The role of reactive oxygen species and metabolism on cancer cells and their microenvironment. Semin Cancer Biol. 2014;25:23-32. doi: 10.1016/j.semcancer.2013.12.007. [PubMed: 24406211].

10. Joseph SK, Booth DM, Young MP, Hajnoczky G. Redox regulation of ER and mitochondrial $\mathrm{Ca}(2+)$ signaling in cell survival and death. Cell Calcium. 2019;79:89-97. doi: 10.1016/j.ceca.2019.02.006. [PubMed: 30889512].

11. Lawson M, Jomova K, Poprac P, Kuča K, Musílek K, Valko M. Free Radicals and Antioxidants in Human Disease. Nutritional Antioxidant Therapies: Treatments and Perspectives. Springer; 2017. p. 283-305. doi: 10.1007/978-3-319-67625-8_12.

12. Villanueva I, Alva-Sanchez C, Pacheco-Rosado J. The role of thyroid hormones as inductors of oxidative stress and neurodegeneration. Oxid Med Cell Longev. 2013;2013:218145. doi: 10.1155/2013/218145. [PubMed: 24386502]. [PubMed Central: PMC3872098].

13. Akinci M, Kosova F, Cetin B, Sepici A, Altan N, Aslan S, et al. Oxidant/antioxidant balance in patients with thyroid cancer. Acta Cir Bras. 2008;23(6):551-4. doi: 10.1590/s0102-86502008000600013. [PubMed: 19030755].

14. Wang D, Feng JF, Zeng P, Yang YH, Luo J, Yang YW. Total oxidant/antioxidant status in sera of patients with thyroid cancers. Endocr Relat Cancer. 2011;18(6):773-82. doi:10.1530/ERC-11-0230. [PubMed: 22002574]. [PubMed Central: PMC3230112].

15. Aycicek A, Erel O. Total oxidant/antioxidant status in jaundiced newborns before and after phototherapy. J Pediatr (Rio J). 2007;83(4):31922. doi: 10.2223/JPED.1645. [PubMed: 17625638].

16. Rahman K. Studies on free radicals, antioxidants, and co-factors. Clin Interv Aging. 2007;2(2):219-36. [PubMed: 18044138]. [PubMed Central: PMC2684512].

17. Ashtekar A, Huk D, Magner A, La Perle KMD, Boucai L, Kirschner LS. Alterations in Sod2-Induced Oxidative Stress Affect Endocrine Cancer Progression. J Clin Endocrinol Metab. 2018;103(11):4135-45. doi: 10.1210/jc.2018-01039. [PubMed: 30165401]. [PubMed Central: PMC6194813].

18. Miri MM, Mohammadi P. [Evaluation of oxidative stress biomarkers in patients with medullary thyroid carcinoma and compared to healthy subjects]. J Mil Med. 2017;19(1):84-90. Persian.

19. Hosseini-Zijoud SM, Ebadi SA, Goodarzi MT, Hedayati M, Ab basalipourkabir R, Mahjoob MP, et al. Lipid Peroxidation and Antioxidant Status in Patients with Medullary Thyroid Carcinoma: A Case-Control Study. J Clin Diagn Res. 2016;10(2):BC04-7. doi: 10.7860/JCDR/2016/17854.7202. [PubMed: 27042443]. [PubMed Central: PMC4800508].

20. Erdamar H, Cimen B, Gulcemal H, Saraymen R, Yerer B, Demirci $H$. Increased lipid peroxidation and impaired enzymatic antioxidant defense mechanism in thyroid tissue with multinodular goiter and papillary carcinoma. Clin Biochem. 2010;43(7-8):650-4. doi: 10.1016/j.clinbiochem.2010.02.005. [PubMed: 20171198].

21. Famil Sagharchian S, Hedayati M, Kazerouni F, Rahimipour A, Shanaki M. Salivary Lipid Peroxidation and Antioxidant Status in the Patients with Papillary Thyroid Carcinoma: A Case-Control Study. Int J Cancer Manag. 2018;11(3). doi: 10.5812/ijcm.9941. 
22. Lassoued S, Mseddi M, Mnif F, Abid M, Guermazi F, Masmoudi H, et al. A comparative study of the oxidative profile in Graves' disease, Hashimoto's thyroiditis, and papillary thyroid cancer. Biol Trace Elem Res. 2010;138(1-3):107-15. doi: 10.1007/s12011-010-8625-1. [PubMed: 20204550].

23. Nozhat Z, Hedayati M, Azizi F. Thyroid Cancer Epidemic: A Peril or an Alarm? Int J Endocrinol Metab. 2015;13(4). e28491. doi: 10.5812/ijem.28491. [PubMed: 26633981]. [PubMed Central: PMC4659334].

24. Fortunato RS, Ferreira AC, Hecht F, Dupuy C, Carvalho DP. Sexual dimorphism and thyroid dysfunction: a matter of oxidative stress? J Endocrinol. 2014;221(2):R31-40. doi: 10.1530/JOE-13-0588. [PubMed: 24578296].

25. Stanley JA, Neelamohan R, Suthagar E, Vengatesh G, Jayakumar J,
Chandrasekaran M, et al. Lipid peroxidation and antioxidants status in human malignant and non-malignant thyroid tumours. Hum Exp Toxicol. 2016;35(6):585-97. doi: 10.1177/0960327115597982. [PubMed: 26270564].

26. Laatikainen LE, Castellone MD, Hebrant A, Hoste C, Cantisani MC, Laurila JP, et al. Extracellular superoxide dismutase is a thyroid differentiation marker down-regulated in cancer. Endocr Relat Cancer. 2010;17(3):785-96. doi: 10.1677/ERC-10-0021. [PubMed: 20576801].

27. Erdamar H, Demirci H, Yaman H, Erbil MK, Yakar T, Sancak B, et al. The effect of hypothyroidism, hyperthyroidism, and their treatment on parameters of oxidative stress and antioxidant status. Clin Chem Lab Med. 2008;46(7):1004-10. doi: 10.1515/CCLM.2008.183. [PubMed: 18605962]. 